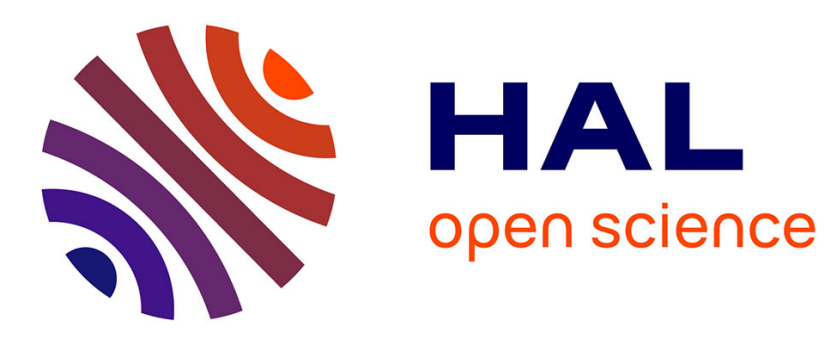

\title{
The case of Textbooks in Mathematics Teaching
}

Christophe Hache

\section{To cite this version:}

Christophe Hache. The case of Textbooks in Mathematics Teaching. Fabrice Vandebrouck. Mathematics Classrooms, Studens' Activities and Teachers' Practices, Sense Publishers, pp.117-133, 2013. hal-00903897

\section{HAL Id: hal-00903897 \\ https://hal.science/hal-00903897}

Submitted on 13 Nov 2013

HAL is a multi-disciplinary open access archive for the deposit and dissemination of scientific research documents, whether they are published or not. The documents may come from teaching and research institutions in France or abroad, or from public or private research centers.
L'archive ouverte pluridisciplinaire HAL, est destinée au dépôt et à la diffusion de documents scientifiques de niveau recherche, publiés ou non, émanant des établissements d'enseignement et de recherche français ou étrangers, des laboratoires publics ou privés. 
CHRISTOPHE HACHE

\section{CHAP 5. LE CAS DES MANUELS DANS L'ENSEIGNEMENT DES MATHEMATIQUES}

\section{INTRODUCTION}

Le manuel de mathématiques est, en France, un objet central dans les pratiques professionnelles des enseignants (et de façon plus marquée encore chez les débutants) et dans le travail quotidien des élèves, que ce soit en classe ou dans leur travail personnel. Il est pourtant paradoxalement peu étudié pour lui-même dans les recherches sur l'enseignement en France (Bruillard, 2005) et notamment dans les recherches en didactique des mathématiques ${ }^{1}$.

Il nous faut tout d'abord souligner qu'en France la conception des manuels est à la charge d'éditeurs privés qui proposent, chacun, aux enseignants leurs manuels. Le choix du manuel donné aux élèves revient donc aux enseignants, le financement est assuré par l'administration.

Un manuel est le résultat d'un jeu complexe de contraintes multiples. Nous nous intéresserons dans une première partie à décrire la perception que nous en avons eu lors d'une expérience d'élaboration et de rédaction d'un manuel. Nous nous efforcerons de mettre en évidence les marges de manœuvre qui en découlent.

Compte tenu de cette description, il nous a paru intéressant d'évaluer l'homogénéité des manuels, de rechercher si les auteurs profitent de ces marges de manœuvres lors de l'écriture, et notamment lors de l'écriture du panel d'exercices qu'ils proposent. Nous avons donc analysé les exercices de quatre manuels (sur un même thème) en fonction des connaissances mises en jeu et des activités qu'ils pourraient engendrer chez les élèves.

\section{CONTRAINTES ET MARGES DE MANEUVRE DANS LA CONCEPTION D'UN MA- NUEL, UN POINT DE VUE ISSU DE L'EXPERIENCE}

Cette première partie tente une description du jeu des contraintes pesant sur l'écriture, et de façon moins centrale sur l'utilisation des manuels. Elle est issue de l'expérience de Christophe Hache comme responsable de la collection Domino lors de la parution des manuels pour la classe de sixième ${ }^{2}$ (Hache, 2005) et de cinquième $^{3}$ (Hache, 2006).

$$
* *
$$

Le manuel dont nous parlons est celui « de l'élève ». Ce livre est donné à l'élève en début d'année et repris en fin d'année. Les contraintes imposées par le fait que, matériellement, le manuel est un livre concernent, évidemment les utilisateurs mais aussi, par leur biais, les concepteurs du manuel.

F. Vandebrouck (ed) Activités des élèves et pratiques des enseignants en classe de mathématique. Ouvrage collectif. 
Citons dans un premier temps, par exemple, le fait que l'élève ait à sa disposition l'ensemble du manuel complique, gêne l'énoncé de toute activité de découverte d'une notion (l'ensemble du chapitre concernant la notion n'est, en effet, jamais loin), la présence de l'ensemble des questions d'un problème peut gêner une réelle recherche lors de la résolution des premières questions. De même la position d'un exercice dans le manuel est une indication de facto quant à sa résolution.

Les enseignants peuvent avoir accès à un «livre du professeur ». Mais si l'on en retire la retranscription des programmes officiels et les corrigés des exercices du manuel, leur contenu s'avère en général pauvre. Au collège ${ }^{4}$, en mathématiques, les enseignants utilisent peu ce manuel du professeur ${ }^{5}$ (les choses sont moins tranchées par exemple au niveau primaire, où le contenu y est d'ailleurs bien plus développé, et où les enseignants ne sont en général pas spécialisés en mathématiques).

Les séances de travail en groupe, les séances de recherches longues, ou nécessitant des modalités de mise en place particulières (échanges entre élèves, débat...) trouvent, de ce fait, difficilement leur place dans les manuels.

La forme papier rend peu naturelle l'appropriation ou la modification tu texte par les enseignants (adaptation à l'approche choisie, adaptation aux élèves etc.). Sur ce point, on peut citer la nouveauté du manuel Sésamath (Sésamath, 2006) : le livre est vendu sous forme papier mais il est aussi disponible intégralement sous forme numérique dans un format permettant toutes modifications (Sésamath, Site).

Une troisième contrainte est liée à la stabilité des pratiques liées aux manuels (écriture, utilisation, manipulation...). Les attentes des enseignants (qui choisissent le manuel), les représentations des auteurs ou des éditeurs sur ce que doit contenir un manuel, sur la façon de le présenter sont fortement influencées par l'omniprésence et l'homogénéité des manuels existants ou passés. On peut, par exemple, citer l'expérience du manuel Sésamath (Sésamath, 2006) qui a été conçu par un collectif important d'enseignants bénévoles sans intervention d'un éditeur. La visée non commerciale du projet et l'absence de l'éditeur lors de l'écriture suppriment un grand nombre de contraintes comme on le verra, le manuel reste cependant en grande partie très classique. Le projet n'était pas de produire un manuel révolutionnaire mais la proximité du résultat avec les standards montre bien une grande stabilité dans les représentations et les attentes.

Enfin, il existe une contrainte de temps dans l'écriture du manuel. Les changements de programmes suivent généralement une classe d'âge : les programmes sont, par exemple, modifiés en 6ème à la rentrée 2005, puis en 5ème à la rentrée 2006 etc. jusque la 3ème en 2008. Ces changements de programmes sont accompagnés du renouvellement des manuels. Les équipes d'auteurs n'étant, en général, pas pléthoriques $^{6}$ elles travaillent sur un seul manuel à la fois. Elles disposent donc d'un an environ pour concevoir les manuels à partir des programmes. Ce délai d'un an est une des contraintes les plus fortes pesant sur la conception du manuel : peu de prise de recul, peu de possibilités de retour en arrière, peu de relecture, très peu 
d'expérimentations, beaucoup de travail dans l'urgence, les collaborations et consultations extérieures sont difficiles à mettre en place...

Les relations entre l'éditeur de manuel scolaire ${ }^{7}$ et les auteurs sont continues : l'éditeur est présent dès le début du travail et suit l'écriture de façon ininterrompue ${ }^{8}$. La structuration du livre, la maquette des chapitres, les rubriques d'exercices... sont autant d'éléments intervenant très tôt dans la conception. Ils relient contenu (sous la responsabilité principale des auteurs) et forme (sous la responsabilité principale de l'éditeur). Le travail des auteurs ne peut donc se faire sans une prise en compte des contraintes de l'édition (et réciproquement). L'éditeur a, de façon centrale, un objectif commercial : il conçoit des produits, recherche des clients (cherche à les séduire face à la concurrence) et vend. La quantité de produits vendus détermine le bénéfice dégagé. On peut par exemple citer le fait qu'il n'existait pas, en 2008, de manuel en terminale ${ }^{9}$ littéraire car le marché est «trop petit». D'un point de vue commercial le «client» est facile à définir : c'est l'enseignant qui, au sein de son équipe et une fois qu'il a reçu tous les manuels édités, doit choisir celui qui sera acheté pour les élèves de l'établissement l'année suivante. Les attentes du «client » sont par contre difficiles à cerner, les critères de choix ne sont ni explicites ni totalement rationnels.

«Les éditeurs recherchent naturellement, en priorité, l'adhésion des enseignants prescripteurs ; les manuels sont donc conçus en fonction des vœux des professeurs plus qu'en fonction des souhaits de l'institution ou des besoins des élèves. (...) Si les éditeurs continuent à proposer, comme ils l'affirment constamment, le produit souhaité par les enseignants et que ces mêmes enseignants choisissent de préférence un produit rassurant qui les conforte dans leurs habitudes, comment faire passer l'innovation pédagogique ? (...) La réflexion sur le manuel et ses nécessaires évolutions doit partir des besoins des élèves et non des vœux des enseignants. » (Borne, IGEN ${ }^{10} 1998$ )

Plaire à un maximum d'enseignants (quelles que soient leurs pratiques) et éventuellement intéresser les élèves (ce qui se traduit par «montrer à l'enseignant que le manuel va intéresser ses élèves ») passe, pour l'éditeur, par un certain nombre de règles. Un exemple: le principe d'élaboration du manuel le plus omniprésent, et ce à tous les niveaux, est celui de la régularité. Pour que le manuel soit «bien fait», pour qu'il soit «facile à utiliser pour les élèves », pour qu'il rassure les enseignants, «il faut» que tous les chapitres soient structurés de la même façon (rubriques, nombre de pages...). L'irrégularité des types de notions abordées n'entrent, par exemple, que très peu en ligne de compte.

Le contenu mathématique des manuels est cadré par les programmes officiels. Ceux-ci sont commentés et mis en relief par les « documents d'accompagnement des programmes» ou les «documents d'applications des programmes ». Notons que les programmes paraissent officiellement en général un an avant leur application et que les documents d'accompagnement ou d'application ${ }^{11}$ paraissent plus tard, souvent après la parution des manuels. Ce ne sont pas les seules contraintes liées aux contenus à enseigner. En effet, les contenus mathématiques imposent des contraintes de présentation et d'organisation; c'est un des objets explorés par les recherches en didactique des mathématiques. Le choix des types de 


\section{CHRISTOPHE HACHE}

tâches proposés, leur déclinaison en exercices, l'équilibre des volumes entre les différents types d'activités attendues sont autant d'éléments très contraints par le contenu abordé.

Le travail sur le contenu est essentiellement à la charge des auteurs. L'éditeur n'exerce son influence souvent qu'indirectement, notamment par la rigidité de la forme ou par la contrainte du temps. Il peut arriver cependant à l'échelle d'un exercice ou, plus rarement, à l'échelle d'un chapitre que l'éditeur refuse une façon de présenter une notion (l'argument des instructions officielles n'a alors que peu de poids par rapport à celui, par exemple, de ce que les enseignants ont l'habitude de faire et de ce qu'ils sont prêts, ou supposés être prêts, ou non, à accepter comme changement).

Les marges de manœuvres des auteurs (et ensuite des utilisateurs) de manuels existent. Lors de l'élaboration du manuel, la mise en place de l'équipe d'auteurs (et de l'équipe de relecteurs), les choix éditoriaux macroscopiques (façon d'aborder les programmes, découpage du livre etc.) sont autant de points pour lesquels les contraintes semblent plus faibles (si ce n'est, toujours, la contrainte de temps sur la réflexion, l'expérimentation etc.).

Les marges de manœuvres lors de l'écriture sont également importantes en ce qui concerne la partie «exercices » du livre. En effet, l'éditeur focalise son attention sur les premières parties des chapitres: activités d'introduction, pages de cours, exercices corrigés... On peut avancer les explications suivantes :

- Les éditeurs se fient en cela aux déclarations des enseignants qui disent porter une grande attention aux pages de cours et aux exercices corrigés (rigueur, accessibilité pour les élèves...) dans le choix de leur manuel ${ }^{12}$,

- Les éditeurs (et les enseignants) disposent de très peu d'outils pour analyser et évaluer un exercice ou un panel d'exercices.

La rédaction des exercices est donc essentiellement cadrée par des contraintes quantitatives: taille des énoncés, nombre d'exercices, parfois par la possibilité d'illustration.

Bien sûr, au delà de la confection du manuel, une marge de manœuvre importante est laissée aux enseignants. Le travail de Sara Arditi (Arditi, 2011) étudie précisément la variabilité des pratiques des enseignants utilisant un même manuel. Les habitudes du métier ne laissent pas beaucoup de choix sur le fait d'acheter ou non un manuel mais on peut noter que certains enseignants n'utilisent pas de manuel ; soit qu'ils produisent eux-mêmes le matériel pédagogique dont ils ont besoin, soit qu'ils utilisent indifféremment les différents manuels ou d'autres ressources à leur disposition et la photocopieuse (Ben Salah Breigeat, 2001). Le choix du manuel est un point important dont les critères sont rarement explicités (il n'existe, par ailleurs, pas de recherche à ce sujet, pas ou peu de formation...). C'est ici que l'enseignant joue son rôle de client pour les éditeurs.

La principale liberté de l'enseignant à propos du manuel reste bien sûr celle de l'organisation de son enseignement. Le mode d'utilisation du manuel est sans doute assez varié. Lors de la conception d'une séance ou d'une suite de séance, l'enseignant s'approprie le manuel, reconstruit ce dont il a besoin à partir de ce qui 
est proposé. Lors de l'enseignement, lorsqu'il utilise le manuel, l'enseignant adapte aussi, consciemment ou non, le contenu du manuel au déroulement de la séance.

$* *$

Plusieurs champs de questions apparaissent à la lumière des points évoqués cidessus. On peut se demander tout d'abord quels sont les effets de ces contraintes : y a-t-il une uniformité forte dans les propositions des manuels ? Peut-on distinguer certaines variations ? On peut avancer la question de ce qui n'apparaît pas dans les manuels du fait de ces contraintes. Une lecture en creux est toujours difficile mais il est toujours possible de confronter de ce point de vue le contenu des manuels aux programmes officiels. La mise en évidence et la description d'une éventuelle uniformité, ou au moins proximité, des manuels pose la question de la caractérisation des manuels.

Compte tenu de ce qui précède on peut se demander dans quelle mesure le manuel peut être un outil efficace de diffusion de résultats de recherches et d'approches nouvelles d'enseignement : quel manuel permettrait une telle transposition? Avec quelle organisation lors de l'écriture ?...

De nombreux questionnements sont mis en avant dans ce qui précède : comment organiser les contenus d'enseignement sur l'année (en tant qu'auteur de manuel, mais aussi, plus largement, pour enseigner en classe) ? Comment analyser les attentes des enseignants lors de leur choix de manuel et, ensuite, lors de leur utilisation? Les pratiques des enseignants sont-elles liées au manuel utilisé ? Quelle utilisation est faite par les élèves, en classe ou hors classe ?...

L'idée que les manuels de mathématiques sont très proches les uns des autres pour un même niveau et une époque donnée est très répandue. Les maquettes, le nombre des chapitres (classiquement entre 15 et 20 chapitres au collège, chacun sur une notion précise), les découpages des chapitres en rubriques (classiquement «activités » 13 , « cours », « exercices corrigés », « exercices d'applications », « exercices d'approfondissement », le tout sur une vingtaine de pages) sont autant de points renforçant cette impression. Par ailleurs, on a vu dans le paragraphe précédent que de nombreux éléments semblent concourir à cette uniformisation.

On pourrait opposer à ces déclarations le fait que les enseignants utilisent essentiellement les manuels comme source d'exercices et que les éléments évoqués cidessus (maquette, nombre de chapitres, découpage des chapitres...) sont essentiellement liés à la forme : le choix d'un manuel pourrait donc garder son sens si l'on s'en tient au fond (choix d'approche des notions, exercices proposés etc.).

Notons tout d'abord que la forme n'est évidemment pas sans lien avec le fond : le découpage en chapitres et l'affectation d'une notion à un chapitre empêche, par exemple, très généralement, la mise en valeur du travail sur plusieurs notions (sauf dans les derniers chapitres, à condition de les avoir abordés dans l'ordre proposé par le manuel). Les changements de cadres deviennent plutôt rares et quand ils existent, ils relient rarement deux notions nouvelles. Il n'est pas rare qu'une fois un chapitre clos, la notion étudiée ne soit évoquée qu'une fois ou deux dans tout le reste de l'ouvrage. Que dire alors de la structuration et de l'organisation des connaissances pour les élèves? 


\section{CHRISTOPHE HACHE}

\section{ANALYSE ET CARACTERISATION DES EXERCICES DE MANUELS}

Nous nous proposons dans la suite de ce chapitre de présenter une méthodologie de travail pour aborder cette question de la proximité des manuels, nous présenterons ensuite certains des résultats obtenus (une présentation plus détaillée de la recherche correspondante peut être lue dans Hache 2008). Nous nous sommes demandé, en nous limitant au panel d'exercices proposés dans des chapitres comparables, à quel point les contraintes décrites dans la première partie de notre chapitre avaient un effet unificateur sur le contenu des exercices des manuels.

Le choix de nous centrer sur les exercices a plusieurs justifications :

- Les enseignants utilisent beaucoup les manuels comme base d'exercices (le manuel distribué aux élèves et les autres manuels dont ils disposent).

- On l'a vu, la rédaction des exercices est une des parties du livre où les contraintes imposées aux auteurs sont les moins fortes, et donc où la diversité sera, a priori, la plus importante.

Nous avons étudié un chapitre donné dans quatre manuels. Les choix méthodologiques (choix des manuels, choix du chapitre étudié, et, on l'a vu, choix d'analyser les exercices dans ce chapitre) ont été faits afin d'augmenter les possibilités d'apparition de différences entre les manuels. La notion étudiée dans les exercices analysés a été choisie selon ce critère : il s'agit de la proportionnalité. C'est une des notions traditionnellement étudiées au collège mais les nouveaux programmes lui donnent une place beaucoup plus centrale que précédemment. Son approche a donc évolué par rapport aux anciens programmes, son étude débute plus explicitement dès la sixième. L'organisation du chapitre (la classification des exercices, par exemple, mais aussi le contenu des exercices) est à « réinventer» pour les auteurs : citons par exemple le produit en croix qui ne sera étudié qu'en quatrième alors qu'il était vu en cinquième. La place de la notion changeant dans les programmes, son traitement dans les manuels ne peut se conformer à des canons pré-établis.

Les manuels choisis sont les suivants : Phare 5ème (Brault et al., 2006), Domino 5ème (Hache, 2006), Transmath 5ème (Malaval, 2006) et Sésamath 5ème (Sésamath Ass., 2006). Là aussi le choix a été fait en essayant de favoriser l'hétérogénéité. Phare est une collection récente dont l'édition 6ème a eu un succès très important auprès des enseignants en 2005. Domino aussi est une collection récente écrite par une équipe d'auteurs composée en partie de chercheurs en didactique des mathématiques (peu de succès auprès des enseignants en sixième). Transmath est une des plus vieilles collections existantes, le livre de 6ème a eu un grand succès en 2005. Sésamath est une collection nouvelle (pas de manuel de sixième en 2005), le manuel est rédigé collectivement par une équipe d'une trentaine d'enseignants, il est disponible gratuitement en ligne (et vendu sous forme papier, l'éditeur n'est pas un éditeur scolaire mais un éditeur de logiciels et de CDRom éducatifs et culturels, la version papier du manuel s'est bien vendue en 2006).

À ce point de notre travail nous avons quatre séries exhaustives d'exercices du chapitre proportionnalité de quatre manuels de cinquième édités en 2006. Nous 
allons maintenant présenter la méthode d'analyse mise en place afin de mieux décrire les proximités, ou au contraire, les différences entre ces manuels.

Nous analysons les tâches proposées dans les manuels en fonction des activités que nous pensons qu'elles engendreront chez l'élève. Pour ce faire, nous nous placerons dans une situation théorique où l'élève maîtrise son cours (en cas de doute sur le contenu de ce cours, ne pouvant nous référer au cours proposé par l'enseignant, nous nous référerons au cours du manuel étudié), où il s'est approprié la question et où il a pour objectif de résoudre l'exercice...

Afin de décrire les activités possibles des élèves à partir d'un énoncé d'exercice, nous avons utilisé la classification des adaptations introduite par Robert (chapitre 2). Pour chaque énoncé étudié nous avons détaillé les différentes adaptations nécessaires pour la réponse envisagée en précisant les connaissances mises en jeu. Pour ce faire, il nous a paru important de préciser et de classer les différentes connaissances utilisées en cinquième à propos de la proportionnalité (qu'elles soient directement liées à cette notion ou utilisées en parallèle).

\section{Analyse d'énoncés : contenu mathématique}

Dans ce paragraphe nous allons détailler la façon dont nous analysons le contenu mathématique à enseigner. Étape préalable au travail d'analyse des exercices.

Extraits des programmes nationaux :

Programmes de sixième 2004 (Bulletin officiel hors série $\mathrm{n}^{\circ} 5 \mathrm{du} 9$ sept. 2004) :

«La résolution de problèmes de proportionnalité est déjà travaillée à l'école primaire. Elle se poursuit en classe de sixième, avec des outils nouveaux »

«Traiter les problèmes "de proportionnalité", en utilisant des raisonnements appropriés, en particulier :

- passage par l'image de l'unité ;

- utilisation d'un rapport de linéarité, exprimé, si nécessaire, sous forme de quotient ;

- utilisation du coefficient de proportionnalité, exprimé, si nécessaire, sous forme de quotient.

Reconnaître les situations qui relèvent de la proportionnalité et celles qui n'en relèvent pas. Appliquer un taux de pourcentage »

Programmes de cinquième 2005 (Bulletin officiel $n^{\circ} 5$ du 25 août 2005) :

«La proportionnalité occupe toujours une place centrale »

«Compléter un tableau de nombre représentant une relation de proportionnalité dont les données sont fournies partiellement. En particulier déterminer une quatrième proportionnelle.

Reconnaître si un tableau complet de nombres est, ou non, un tableau de proportionnalité.

Mettre en oeuvre la proportionnalité dans les cas suivant :

- comparer des proportions,

- calculer et utiliser un pourcentage, 


\section{CHRISTOPHE HACHE}

- calculer et utiliser l'échelle d'une carte ou d'un dessin,

- reconnaître un mouvement uniforme à l'existence d'une relation de proportionnalité entre durée et distance parcourue, utiliser cette proportionnalité »

Les connaissances étudiées en cinquième ont été, au travers du filtre des manuels, classifiées comme suit.

\section{Reconnaissance d'une situation / d'un tableau de proportionnalité}

Beaucoup de choses restent, dans les manuels, de l'ordre du savoir caché dans ce domaine. Lorsque c'est le cas, le fait que la situation est une situation de proportionnalité par choix, par modélisation, afin de pouvoir mener des calculs est notamment toujours implicite. Ceci va gêner dans quelques énoncés où la proportionnalité est admise alors qu'elle pourrait tout aussi bien être questionnée (ce qui est en général fait dans un ou deux exercices par manuel).

Exemple de situation où la proportionnalité ne va pas de soi :

Quelle distance parcourt en 2 h 30 min un escargot qui rampe à la vitesse de $14 \mathrm{~m}$ par heure. (Transmath, $\left.\mathrm{n}^{\circ} 56 \mathrm{p} 99\right)^{14}$

Exemple de remise en cause :

«En ce moment, la durée du jour (entre le lever et le coucher du soleil) diminue de 3 min par jour. De quelle durée aura-t-elle diminué au bout de 30 jours ? Q Que sous-entend cet énoncé ? A-t-il raison ou tort et pourquoi ? (Transmath, $\mathrm{n}^{\circ} 46 \mathrm{p} 99$ )

De même la distinction entre situation de proportionnalité et tableau de proportionnalité est parfois floue :

Un escargot se déplace sur une branche.

La durée de son déplacement, en minutes, et la distance qu'il a parcourue, en centimètres, sont données dans le tableau suivant.

\begin{tabular}{|c|c|c|c|}
\hline Durée (en min) & 4 & 6 & 9 \\
\hline Distance (en cm) & 26 & 39 & 58,5 \\
\hline
\end{tabular}

1) a. Montrer que le mouvement que suit cet escargot paraît uniforme.

b. Préciser le coefficient de proportionnalité de cette situation.

2) $(\ldots)$

b. Quelle distance peut parcourir l'escargot en une heure si son mouvement reste uniforme ? (...) (Phare, $\left.\mathrm{n}^{\circ} 70 \mathrm{p} 130\right)$

L'utilisation du verbe " paraître » semble indiquer que l'énoncé souligne le fait que le caractère proportionnel du tableau ne permet pas de prouver le fait que le mouvement est uniforme. Les deux questions $b$. semblent sous entendre l'inverse. De même :

Pour les exercices 14 à 19, dire si le tableau correspond à une situation de proportionnalité. 
Si oui, préciser le coefficient de proportionnalité. (...) (Phare, p124)

Nous avons nommé cinq connaissances relatives à la mise en évidence d'une situation de proportionnalité (ou d'un tableau) :

- Savoir reconnaître l'existence d'une situation de proportionnalité, ou la forte présomption d'existence (codé Rr) ;

- Savoir trouver (ou proposer) les grandeurs proportionnelles (codé Rg) ;

- Savoir admettre la preuve de la proportionnalité (c'est le cas dans de nombreuses situations concrètes) (codé Ra) ;

- Savoir prouver la proportionnalité (calcul des quotients pour un tableau, existence d'une formule dans une situation, existence d'un mouvement uniforme, d'une échelle etc.) (codé Rp) ;

- Savoir prouver la non-proportionnalité (codé Rnp).

Exemple :

Sur l'étiquette d'une bouteille d'un litre de jus de fruit on lit :

$\begin{array}{ll}\text { Valeurs nutritionnelles moyennes } \\ \text { Protéines } & 0,4 \mathrm{~g} / 100 \mathrm{~mL} \\ \text { Glucides } & 11,8 \mathrm{~g} / 100 \mathrm{~mL} \\ \text { Lipides } & <0,1 \mathrm{~g} / 100 \mathrm{~mL} \\ \text { Valeur énergétique moyenne }: 50 \mathrm{Kcal}\end{array}$

Recopie puis complète le tableau suivant :

\begin{tabular}{|l|c|c|c|c|}
\hline Volume de jus d'orange & $1 \mathrm{~L}$ & $0,25 \mathrm{~L}$ & $1,5 \mathrm{~L}$ & $2 \mathrm{~L}$ \\
\hline Protéines & & & & \\
\hline Glucides & & & & \\
\hline Lipides & & & & \\
\hline Valeur énergétique & & & & \\
\hline
\end{tabular}

(Sésamath, n5 p76)

Cet exercice nécessite entre autres de reconnaître dans cette situation plusieurs situations de proportionnalité ( $\mathrm{Rr}$ ), les cas des lipides (borne supérieure) et de la valeur énergétique $(50 \mathrm{Kcal} / 100 \mathrm{~mL}$ ou $50 \mathrm{Kcal} / \mathrm{L}$ ?) sont particulièrement délicats, ils pourraient être codés Rg. Dans tous les cas la proportionnalité est finalement admise (Ra). Les deux dernières catégories sont liées aux preuves.

\section{Exploitation d'une situation de proportionnalité}

La proportionnalité étant donnée, admise ou prouvée, diverses exploitations peuvent être envisagées. Nous avons distingué les cas suivants :

- Le coefficient (ou échelle, ou vitesse, ou pourcentage...) étant connu, ainsi qu'une seule donnée, il s'agit de savoir calculer la donnée manquante.

o $[\mathrm{Cmu}]$ On multiplie par le coefficient

${ }^{\circ}$ [Cdi] On divise par le coefficient

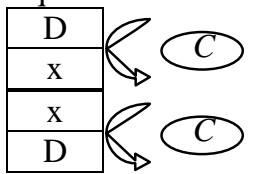

- De même, une formule étant donnée, savoir l'utiliser :

$\circ$ Fmu $]$ directement, 


\section{CHRISTOPHE HACHE}

○ [Fdi] en commençant par l'inverser.

L'utilisation du coefficient seul apparaît très rarement dans les manuels. L'utilisation des formules n'est pas envisagée. C'est donc sur les trois connaissances suivantes que se concentre le travail :

- [Coef] Deux données sont connues, savoir calculer le coefficient

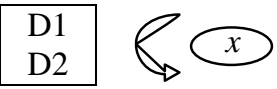

- [QP] Quatrième proportionnelle. Trois données sont connues, savoir calculer la quatrième. En général plusieurs méthodes sont possibles: calcul (puis utilisation) du coefficient, retour à l'unité, multiplication d'une «colonne» pour obtenir

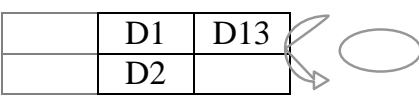
l'autre (la méthode peut être imposée ou non).

- [DonInc] Données à compléter : les données connues ne sont pas en nombre juste suffisant pour calculer la donnée manquante (soit qu'il y ait «trop » de données, soit qu'il y ait plusieurs données manquantes). Une connaissance nouvelle apparaît par exemple : le fait de savoir utiliser l'addition de «colonnes » (la méthode peut être imposée ou non).

\section{Autres connaissances travaillées}

Dans les chapitres travaillant la notion de proportionnalité d'autres connaissances sont utilisées ou sont travaillées. Nous avons retenu les groupes de connaissances suivants :

- Graphiques, tableaux

La proportionnalité est travaillée en sixième (et avant) à travers l'idée de proportionnalité entre deux grandeurs. Les tableaux et les graphiques sont aussi des outils travaillés en sixième (lecture et interprétation, quelques constructions sont envisagées). En cinquième on introduit le tableau de proportionnalité et son lien avec les situations de proportionnalité déjà étudiées.

Le lien entre l'alignement de points avec l'origine d'un repère et la proportionnalité peut être vu sans que ce soit exigible, il sera étudié explicitement en quatrième. Il existe par contre un lien fort entre représentations graphiques de données et proportionnalité (proportionnalité entre effectif et hauteur pour un diagramme en bâton, entre effectifs et angle dans un diagramme circulaire etc.).

- Connaissances liées aux grandeurs

Nous avons distingué les connaissances sur les conversions de diverses grandeurs (longueurs, aires, masses, volumes, durées, vitesse), des connaissances liées à la notion de grandeur elle-même (reconnaître la grandeur en jeu, comparaison de grandeurs sans mesure ou avec une unité ad hoc).

Sur ce point, notons que les premières grandeurs et leurs mesures étudiées le sont dès l'école primaire (longueur, masse, contenance et durée sont, par exemple, abordés au cycle 2), les angles et les volumes sont abordés au cycle 3 et leur mesure est étudiée en sixième. Les conversions de mesures de volumes sont des connaissances en cours d'acquisition en cinquième.

La vitesse a un statut particulier : elle n'est pas étudiée en tant que telle, seule l'idée de «vitesse uniforme » est abordée (comme un cas de relation de proportionnalité 
entre durée et longueur). Les grandeurs quotients sont introduites par le programme de quatrième.

- Connaissances liées au contexte

De nombreuses connaissances sont utilisées dans la résolution d'exercices liés à la proportionnalité. En plus de celle citées ci-dessus (liées aux grandeurs, aux graphiques ou aux tableaux) de nombreuses connaissances anciennes liées à la géométrie sont utilisées (essentiellement des tracés, des mesures, des formules, éventuellement en géométrie dans l'espace). De même on manipule les arrondis, les ordres de grandeurs, la notation fractionnaire, les pourcentages (notions étudiées dès la sixième).

- Autres

Quelques exercices tentent de faire le lien entre le travail de la proportionnalité et le début des manipulations algébriques en cinquième. Un manuel propose quelques exercices utilisant la notation scientifique des nombres, c'est une connaissance étudiée en quatrième.

\section{Analyse d'énoncés : adaptations}

Ces adaptations sont regroupées en huit catégories (voir chapitre 2) :

- TSI : tâches simples et isolées, il s'agit de tâches pour lesquelles l'élève, lors de la résolution envisagée, utilisent une connaissance sans adaptation. Cf. exemples 1, 2 et 3 .

- A1 : l'élève doit, pour pouvoir utiliser une connaissance, reconnaître les modalités de son application. Cf. exemples 2, 6 et 8 .

- A2 : l'élève doit introduire un intermédiaire pour avancer dans son travail. Il peut avoir à placer un nouveau point en géométrie (voire simplement à nommer un point existant), faire un calcul intermédiaire etc. Cf. exemples 3, 4 et 5.

- A3 : il s'agit ici de mettre en relations plusieurs connaissances, de faire un changement de cadre, de relier le travail sur des connaissances nouvelles avec des connaissances anciennes. Cf. exemples 3 et 6 .

- A4: L'énoncé laisse à la charge de l'élève l'introduction d'une étape du raisonnement. Il n'est pas rare, par exemple, dans les exercices relatifs à la proportionnalité utilisant un contexte que l'hypothèse de la proportionnalité de la situation soit laissée sous silence, c'est alors à l'élève, théoriquement, de le décider ou de le supposer. Cf. exemple 7.

- A5 : La réponse à une question nécessite l'utilisation d'un résultat établi dans les questions précédentes.

- A6 : Choix d'une méthode entre plusieurs. Cf. exemple 8.

- A7 : travail sur des connaissances non encore introduites en tant que telles à ce niveau, non encore formalisées. Cf. exemples 9.

Exemple 1 (il s'agit d'un QCM) :

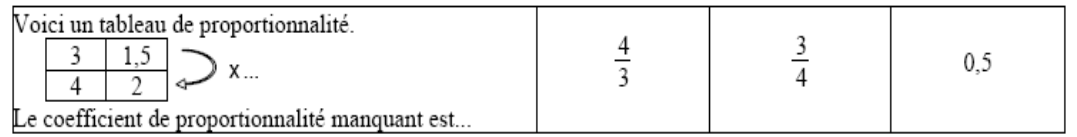

(Transmath, ${ }^{\circ} 60 \mathrm{p} 100$ ) 


\section{CHRISTOPHE HACHE}

La proportionnalité est posée explicitement par l'énoncé. La question est précise (calcul du coefficient) et fait référence à un point du cours. On peut penser que l'absence de contexte permet une application du cours sans difficulté particulière. La question a été codée TSI-Coef.

Exemple 2:

Le prix d'une paire de lunettes de soleil est augmenté de 3,20€.

Son prix initial était de $40 €$.

A quel pourcentage du prix initial correspond cette augmentation? (Phare, $\left.\mathrm{n}^{\circ} 3 \mathrm{p} 122\right)$

Les données numériques nécessaires sont dans l'énoncé, l'élève doit cependant adapter ses connaissances à la situation posée. La question a été codée A1-QP (il a en effet été décidé de coder les calculs de pourcentage QP : ils sont présentés en cinquième comme un calcul de quatrième proportionnelle).

La distinction entre TSI et A1 est faite en fonction du contexte. L'existence d'un habillage, l'existence d'exemples proches de la situation de l'exercice dans le cours, la place de l'exercice dans le chapitre sont autant d'éléments qui peuvent être pris en compte pour décider de la qualification de l'adaptation nécessaire à l'élève.

Exemple 3:

1er exemple: Donner une échelle possible d'une maison de poupée qui mesure $50 \mathrm{~cm}$ de haut. (Domino, $\mathrm{n}^{\circ} 39 \mathrm{p} 63$ )

Il est ici nécessaire d'introduire l'ordre de grandeur de la taille d'une maison (telle qu'on se l'imagine), ce qui a été codé A2-Ordre de grandeur. Deux autres activités sont codées pour cet exercice : le changement d'unité (A3-Conversion de longueur) et le calcul de l'échelle une fois la hauteur réelle choisie et les conversions effectuées (TSI-Coef).

Exemple 4 :

1. Représenter par un diagramme circulaire la répartition de la population par continent en 2003.

2. Quelles données supplémentaires permettraient de compléter l'étude?

(Domino, $\left.\mathrm{n}^{\circ} 45 \mathrm{p} 64\right)$
Répartition de la population par continent en 2003

\begin{tabular}{|c|c|}
\hline Continent & Pourcentage \\
\hline Afrique & $13,6 \%$ \\
\hline Amérique & $13,7 \%$ \\
\hline Asie & $60,7 \%$ \\
\hline Europe & $11,5 \%$ \\
\hline Océanie & $0,5 \%$ \\
\hline
\end{tabular}

Pour résoudre la question 1), l'élève doit introduire des données numériques : sous une forme ou sous une autre il doit introduire une ligne «Total». Cette partie de la question est codée A2-Total.

De même dans l'exercice ci-dessous, c'est à lui, dans la question 1), de décider une mesure à effectuer (cette partie de la question a été codée A2-Mesures).

Exemple 5:

Un plan d'appartement est donné, les longueurs a, b, c et d y sont codées. La longueur $a$ est en réalité $17,2 \mathrm{~m}$. 
1) Déterminer l'échelle de ce plan.

2) Déterminer les longueurs réelles $b, c$ et $d$. (Phare, $\left.{ }^{\circ} 8 \mathrm{p} 123\right)$

\section{Exemple 6:}

Lors d'une traversée de l'Atlantique à la voile, le skipper a noté et relevé ses caps pour pouvoir tracer ensuite la route qu'il a empruntée.

Une ligne brisée est tracée (6 segments, «Boston » à gauche, «Lisbonne » à droite)

a. Quelle est, au millimètre près, la longueur de la ligne brisée qui représente sa route.

b. Son tracé est à l'échelle 1/600 000 000. Quelle distance a-t-il parcourue ?

(Sésamath, $\mathrm{n}^{\circ} 14 \mathrm{p} 77$ )

L'élève verra sans doute l'utilité d'un changement d'unité de longueur pour répondre à la question $b$., les conversions de longueurs sont des connaissances anciennes (revues en sixième), cette partie de la question est codée A3-Conversion de longueur. L'utilisation de l'échelle dans la question b. a été codée A1-Cdi. Notons que c'est une des rares fois dans le panel d'exercices étudié que le coefficient est utilisé avec une seule donnée (Cdi ou Cmu).

\section{Exemple 7 :}

Dans un marais salant il faut faire évaporer $1000 \mathrm{~g}$ d'eau de mer pour obtenir $32 \mathrm{~g}$ de sel.

a. Calculer la masse de sel obtenue à partir de $500 \mathrm{~kg}$ d'eau de mer.

b. Quel poids d'eau de mer doit s'évaporer pour obtenir une tonne de sel ? (Transmath, $\mathrm{n}^{\circ} 17 \mathrm{p} 96$ )

Même s'il ne fait pas de doute que le modèle choisi ici par exemple est la proportionnalité, c'est une part du travail de l'élève de le signaler (et éventuellement de s'en persuader), cette partie du travail a été codée A4-Rr.

Exemple 8:

On donne les correspondances suivantes concernant les unités anglosaxonnes :

\begin{tabular}{|l|l|}
\hline Pour les longueurs : & Pour les masses : \\
1 yard $=0,9144 \mathrm{~m}$ & 1 pound (livre) $=16$ ounces \\
1 foot (pied) $=12$ inches (pouces) & 1 pound $=0,4536 \mathrm{~kg}$ \\
1 yard $=3$ feet (pieds) & \\
1 mille $=1760$ yard & \\
\hline $\begin{array}{l}\text { Exprimer la taille et la masse des élèves de la classe dans ces unités anglo- } \\
\left.\text { saxonnes. (Domino, } \mathrm{n}^{\circ} 69 \mathrm{p} 67\right)\end{array}$
\end{tabular}

C'est ici à l'élève de mettre en place sa méthode de travail pour trouver les unités adaptées à la situation et pour effectuer les calculs correspondants. Cette partie de la question a été codée A6-Grandeur.

Notons, là aussi, que la qualification en A6 peut dépendre du contexte. Il se peut qu'à l'intérieur d'un raisonnement un élève ait à faire des choix de méthode, l'adaptation peut être classée A1 par exemple si ce choix est très local et est 


\section{CHRISTOPHE HACHE}

explicité et détaillé dans le cours. Lorsqu'un tableau de proportionnalité incomplet est donné, il existe, par exemple, plusieurs méthodes pour le compléter (calcul du coefficient, addition de colonnes etc.) ce type d'étape a en général été classé A1. Exemple 9:

La vitesse du son est de 340 mètres par seconde et celle de la lumière est de 299792480 mètres par secondes.

a. Exprime ces vitesses en kilomètres par heure.

(...) (Sésamath, $\mathrm{n}^{\circ} 28 \mathrm{p} 78$ )

La notion de vitesse et les changements d'unités de mesure correspondant sont vues en quatrième (codé A7-Vitesse).

\section{CONCLUSIONS}

Dans le travail évoqué ici (Hache, 2008) nous avons testé, dans le cadre de recherche en didactique des mathématiques, certaines des contraintes et des marges de manœuvre ressenties lors de la conception d'un manuel scolaire. Nous avons notamment décrit la méthodologie mise en place pour tester la proximité des panels d'exercices proposés dans quatre manuels français (pour le chapitre proportionnalité en cinquième). À cette fin nous avons analysé chaque exercice de manuel en détaillant les différentes adaptations nécessaires à sa résolution en les mettant en lien avec les connaissances mathématiques travaillées.

Les conclusions peuvent être de plusieurs ordres. Nous pouvons tout d'abord nous demander si les analyses faites confortent l'impression d'uniformité dans le contenu des manuels pour le lecteur et, par ce biais, interroger l'effectivité des contraintes ressenties lors de la conception. Nous pouvons par ailleurs conclure sur le traitement de la proportionnalité dans les manuels : les analyses faites permettent de mettre en évidence le type d'activités proposées aux élèves... et certaines des activités qui ne sont pas proposées.

Avant de conclure sur ces points il nous faut souligner la distance quil y a entre l'analyse des énoncés d'un manuel et les activités des élèves en classe. Trois filtres importants s'interposent entre les deux :

- l'enseignant, lorsqu'il prépare sa séance, en supposant qu'il choisisse des exercices pour ses élèves uniquement dans le manuel envisagé, ne va pas tous les traiter. Les analyses quantitatives regroupant l'ensemble des exercices n'ont alors plus qu'un sens restreint, une valeur indicative: cet ensemble d'exercices correspond à l'offre proposée aux enseignants, l'offre sera plus riche pour certain type d'activités possibles pour les élèves et moins riche pour d'autres. L'enseignant pourra (ou non) nuancer, dans une certaine mesure, ces importances relatives lors de son choix d'exercices.

- L'enseignant peut, par ailleurs, modifier un énoncé. Il peut le faire consciemment et a priori, avant de le proposer à ses élèves, mais il le fera surtout (et nécessairement) lors du déroulement de la séance. On voit dans d'autres chapitres de ce livre que ces modifications de la tâche proposée vont souvent dans le sens d'une simplification et d'un amoindrissement des adaptations laissées à la charge des élèves. Voir aussi le travail de Sara Arditi sur ce point (Arditi, 2011). 
- Enfin, l'étude de la distance entre l'activité possible inférée de l'analyse d'un énoncé et l'activité effective d'un élève en classe lors du travail sur cet énoncé est un champ de recherche seulement en cours de développement.

Les exercices analysés montrent cependant des tendances lourdes et récurrentes dans le traitement de la notion choisie. On pourrait donc se permettre d'en déduire quelques éléments quant à l'enseignement de la proportionnalité. L'objectif de l'étude était ici plus restreint et consistait à étudier le contenu proposé par les manuels et à en estimer la proximité d'un manuel à l'autre.

Globalement, même si le «portrait » global des manuels laisse peu de place à des fluctuations (les tendances constatées sont très marquées), on peut observer quelques spécificités pour chaque manuel.

On peut noter tout d'abord qu'une part importante (souvent plus de la moitié ${ }^{15}$ ) des activités possibles des élèves sont sur des tâches simples et isolées, aucune adaptation n'est nécessaire à l'utilisation de la connaissance envisagée. Si l'on ajoute les utilisations où l'élève doit «simplement» reconnaître les modalités d'application on s'aperçoit que dans près des trois quarts des cas les connaissances sont utilisées sans laisser de réelle initiative à l'élève: le travail nécessaire à l'apprentissage est donc essentiellement perçu comme une utilisation des connaissances sans réelle prise de recul. Ce phénomène est très stable sur trois des manuels et moins marqué sur l'un d'entre eux (Sésamath) pour lequel des étapes de raisonnement sont, par exemple, à la charge des élèves (il s'agit, en fait, dans la plupart des cas d'introduire l'idée qu'il y a bien proportionnalité).

On note par ailleurs que les connaissances relatives à la proportionnalité utilisées dans les exercices sont surtout liées à l'exploitation d'une situation de proportionnalité (calcul de données manquantes) et sont peu liées au questionnement du caractère proportionnel de la situation (le rapport est d'environ deux tiers, un tiers). Là aussi un des manuels (Domino) est décalé : plus de la moitié des items sont liés à la question de la proportionnalité et non à l'exploitation de la situation de proportionnalité.

Dans ces chapitres sur la proportionnalité, de nombreuses connaissances non directement liées à la proportionnalité sont utilisées: connaissances liées aux mesures de grandeurs, aux graphiques, aux tableaux, à la géométrie, à l'écriture des nombres etc. Globalement la part des connaissances liées à la proportionnalité est majoritaire, ce phénomène se retrouve dans trois des manuels. Dans l'un des manuels (Domino) on trouve une part plus importante $(66 \%$, au lieu de $38 \%$ pour les trois autres manuels) d'activités possibles utilisant des connaissances non directement liées à la proportionnalité.

Les connaissances non directement liées à la proportionnalité varient d'un manuel à l'autre en fonction des situations exploitées. Les contextes des exercices, les outils préconisés, les connaissances utilisées diffèrent (échelles, vitesses uniformes, pourcentages, représentations graphiques de données...).

$\mathrm{Au}$ delà de ces variations, qui permettent de laisser penser que la marge de manœuvre laissée aux auteurs est exploitée a minima ${ }^{16}$, le portrait global du travail sur la proportionnalité dans les manuels est très typé : un travail plutôt calculatoire qu'exploratoire et des exercices presque systématiquement guidés (l'élève n'ayant à 


\section{CHRISTOPHE HACHE}

prendre que très ponctuellement des initiatives). Même si on constate globalement une relative variété des notions abordées au détour du travail sur la proportionnalité, pour chaque manuel les connaissances travaillées sont peu nombreuses (voire très peu nombreuses pour Phare et Sésamath)... On perçoit au travers de ces analyses une absence relative d'exercices permettant à l'élève de s'approprier la question de la proportionnalité pour une situation donnée, de prendre l'initiative du choix des calculs à effectuer, de la méthode à suivre, ou des autres connaissances à mobiliser.

Certaines questions se posent avec plus d'acuité à l'issu de ce travail. Les résultats dégagés sont-ils généralisables ? Notamment, le faible nombre de situations laissant à l'initiative de l'élève quelques adaptations serait inquiétant quant à la réalité et à la nature des apprentissages possibles à partir de ces exercices. Une étude sur plusieurs contenus permettait d'avancer sur ce point.

Il nous semble par ailleurs fondamental de compléter les analyses ci-dessus dans deux directions : les choix des enseignants et les modifications apportées nuancentils les conclusions ci-dessus ? Dans quel sens ? De quelle façon, le cas échéant, le travail des élèves sur les énoncés, en classe ou à la maison, compense-t-il les manques évoqués?

Christophe Hache

Laboratoire de didactique André Revuz.

Université Paris Diderot

${ }^{1}$ Nous pouvons cependant citer la thèse de Ben Salah Breigeat (2001) centrée sur la mise en place des pratiques des jeunes enseignants, elle analyse, entre autre, la proximité entre leur discours et celui du manuel utilisé. Le travail de Robert et Robinet (1989) étudie les représentations méta-cognitives des auteurs de manuels au travers des exercices qu'ils proposent. Parallèlement, l'APMEP (Association des professeurs de mathématiques de l'enseignement public) a encadré de nombreuses réflexions autour de l'objet manuel.

${ }^{2}$ Principalement des élèves de 11 ans.

${ }^{3}$ Principalement des élèves de 12 ans.

${ }^{4}$ Établissement regroupant les classes de 6ème, 5ème, 4ème et 3ème (principalement des élèves de 11 à 14 ans).

${ }^{5}$ On peut par exemple considérer les chiffres de vente de ces manuels lorsqu'ils sont proposés séparément du manuel de l'élève

${ }^{6}$ Le manuel Sésamath se distingue puisqu'il est conçu par un collectif d'une cinquantaine d'enseignants (en ce qui concerne le manuel de 5ème). Plusieurs équipes travaillent en parallèle et le délai d'élaboration du manuel est pour l'instant d'environ deux ans. À noter aussi que la version électronique du manuel (téléchargeable) peut théoriquement continuer à être modifiée après la parution du manuel papier.

${ }^{7}$ Le mot «éditeur » restera ici relativement flou. Il désigne à la fois une entreprise, les personnes qui y travaillent, y compris, mais pas exclusivement, celles qui travaillent sur un manuel de mathématiques..

${ }^{8}$ Notons cependant que dans les manuels commercialisés au collège actuellement deux se distinguent de ce point de vue : le manuel Sésamath, l'éditeur participe peu à la conception, et le manuel Aventure math (Busser et Massot, 2006) conçu et édité par l'équipe d'une revue (Tangente).

${ }^{9}$ Principalement des élèves de 17 ans

${ }^{10}$ Inspection Générale de l'Éducation Nationale. 
${ }^{11}$ Documents officiels complétant, expliquant, enrichissant les textes des programmes.

${ }^{12}$ Paradoxalement les enseignants déclarent, dans le même temps, se servir essentiellement des listes d'exercices dans leur pratique quotidienne.

${ }^{13}$ Le mot «activité » désigne très généralement dans les manuels les exercices à faire avant l'énoncer du cours pour introduire celui-ci : révisions, exercices d'approches, de découverte etc. Cette acception est différente de celle du cadre théorique du présent l'ouvrage.

${ }^{14}$ Pour plus de simplicité, nous nommerons les manuels par leur nom de collection (et non par le nom de leur auteur), nous n'indiquerons pas qu'il s'agit de la classe de cinquième, ni de l'année 2006, sauf dans le cas contraire.

${ }^{15}$ Pour des données plus quantitatives voir Hache 2008.

${ }^{16} \mathrm{La}$ notion et les manuels choisis l'ont été de façon à rendre maximal les variations entre manuels (voir plus haut, le paragraphe sur la méthodologie). 